\title{
Development and Status of the Pierre Auger Observatory
}

\author{
Helio Nogima* \\ Instituto de Física "Gleb Wataghin"/DRCC \\ Caixa Postal 6165, CEP 13081-970, Campinas, SP, Brasil
}

Received on 29 March, 2002

\begin{abstract}
We discuss the status of the Pierre Auger Observatory. The construction of the southern site in Argentina has started a year and a half ago. The project is in its first phase which consists in the construction and running of the so-called engineering array, a prototype of the experiment. The goals, design and construction of the observatory are discussed.
\end{abstract}

\section{The most energetics cosmic rays}

The study of the extremely high energy cosmic rays (EHECR) is difficult due to its low flux - less than 1 particle $\times \mathrm{km}^{-2} \mathrm{~S}^{-1}$ for energies greater than $6 \times 10^{18} \mathrm{eV}$. However the existence of particles with energies above $10^{20} \mathrm{eV}$ has been reported by more than 10 events collected in the last decades by experiments around the world (Haverah Park [1], AGASA [2][3], Fly's Eye [4] - HiRes [5]). There are two main problems that arises from this fact: How to explain the origin of such powerfull radiation and how it propagates through interstellar/intergalatic medium? There are different model categories to explain the origin of the EHECR: one of them supposes that a lower energy cosmic ray is accelerated by Fermi-like mecanism [7] to get up to EHE range (bottom-up). In those theories, though, the relation between the gyro radius of the accelerated particle and magnetic field where the acceleration takes place restricts the objects which could produce $10^{20} \mathrm{eV}$ scale radiation [6]. The known candidates can be summarized as being active galatic nuclei, neutron stars, radiogalaxies and gamma ray bursts. The other models category supposes that the high energy cosmic rays come from the decay of superheavy particles (top-down). The average life of these particles and the amount of them should be consistent with the observed flux of the radiation. Whether or not EHECR origin is connected to some others current cosmological problems is still an issue to be understood.

One of the most interesting aspects of the high energy cosmic rays is on its propagation through the space. Besides magnetic fields, it is subject to the interaction with cosmic microwave background radiation (CMBR). A proton radiation would lose energy interacting with the CMBR photoproducing pions. The less energetic emerging hadron may also interact, again degrading the energy and so on, until the energy gets below the threshold of photoproduction. This leads to the phenomenon predicted by Greisen, Zatsepin and Kuz'min, the so called GZK cutoff effect [8] [9]. The GZK cutoff makes the universe opaque to nucleon radiation for distances longer than $20 \mathrm{Mpc}$ for energies of the order of $2 \times 10^{20} \mathrm{eV}$. For such energies the magnetic field (galatic and intergalatic) should not bend the particle trajectory enough, allowing to point out its source. However, the present data does not seem to indicate any known astrophysical objects in this distance limit. Despite low statistics, it looks isotropic. The goal of the PAO is to increase substantially the statistics and yield data that can help to better understand the GZK effect and the EHECR.

\section{The Pierre Auger Observatory}

Due to the small flux it is necessary to cover large area to observe the EHECR. The collection area must be compatible to the exposure time expected for the experiment. The Pierre Auger Observatory will be consisted of two sites: one at the northern and another at the southern hemisphere. Each one will cover $3000 \mathrm{~km}^{2}$ which, second available data and supposing anisotropy of the EHECR, will collect 30 events above $10^{20} \mathrm{eV}$ per year. For the detection of the extensive air shower (EAS) produced by the EHECR, two differents techniques are employed: ground based particle detectors and fluorescence light detector. The first one consists of sampling different parts of the EAS, measuring the density of particles that hits the ground. This is done spreading several detector stations in a large

\footnotetext{
${ }^{*}$ for the Auger Collaboration
} 
area. From the reconstruction of the lateral distribution function (Eq. 1) the total number of particles of the shower can be obtained, and then the shower energy estimation. Eq. 2 is for $r>800 \mathrm{~m}$ and $E>1 \times 10^{19} \mathrm{eV}$.

$$
\begin{array}{r}
\rho(r)=k r^{-(\eta+r / 4000)}, \\
\eta=3.49-1.29 \sec \theta+0.165 \log \left(\frac{E}{10^{17} e V}\right) . \\
\rho(r)=k r^{-(\eta+r / 4000)}\left(\frac{r}{800}\right)^{1.03} .
\end{array}
$$

The direction of the shower (that must closely match the direction of the EHECR) can be defined by the front shower plane, reconstructed using the time recorded by each detection station - time of flight method.

In the fluorescence technique the detection is made by the fluorescence light yielded by the atmospheric nitrogen when the charged particles from EAS passes through it. The fluorescence isotropic emission allows to observe the shower transversaly to its evolution direction. In this way, by mean of an imaging detector it is possible to scan the developing EAS and get a static image of the cascading process. The geometrical reconstruction of the shower can be done using the time information of each image pixel of the shower. The first step for this is to find the shower detector plane, defined as the plane having the detector center and the axis of the shower (Fig. 1). The relation between the time of each pixel and the angle $\theta$ is given by:

$$
t\left(\theta_{i}\right)=\frac{R_{p}}{c} \tan \left(\frac{\theta_{i}}{2}\right),
$$

where $t\left(\theta_{i}\right)$ is the time registered by the pixel $i$ at the angle $\theta_{i}$. The best fit of the parameters in Eq. 3 gives the shower axis. Knowing the number of photons emmited by each segment of the registered track, the longitudinal profile of the shower can be reconstructed. The depth of the maximum development of the shower can then be identified, what brings information about the chemical composition of the cosmic ray that induced it [10]. The relation between the number of photons and charged particles is given by:

$$
N_{e}=\frac{4 \pi R^{2} N_{p e}}{F_{y i e l d} A \epsilon \xi(R)},
$$

where $R$ is the distance to the corresponding shower track segment, $A$ the light collecting area, $\epsilon$ the detector efficiency and $l$ the track segment length. Knowing the fluorescence yield $F_{\text {yield }}$ in air and the current light transmitance $\xi$ of the atmosphere it is possible to obtain the total number of (charged) particles in the shower $N_{e}$, and then the energy of the cosmic ray by:

$$
E=\frac{E_{c}}{X_{0}} \int N_{e}(x) d x
$$

where $E_{c}$ is the critical energy of the electron $(81 \mathrm{MeV})$ and $X_{0}$ the radiation length in air $\left(37.1 \mathrm{gcm}^{-2}\right)$.

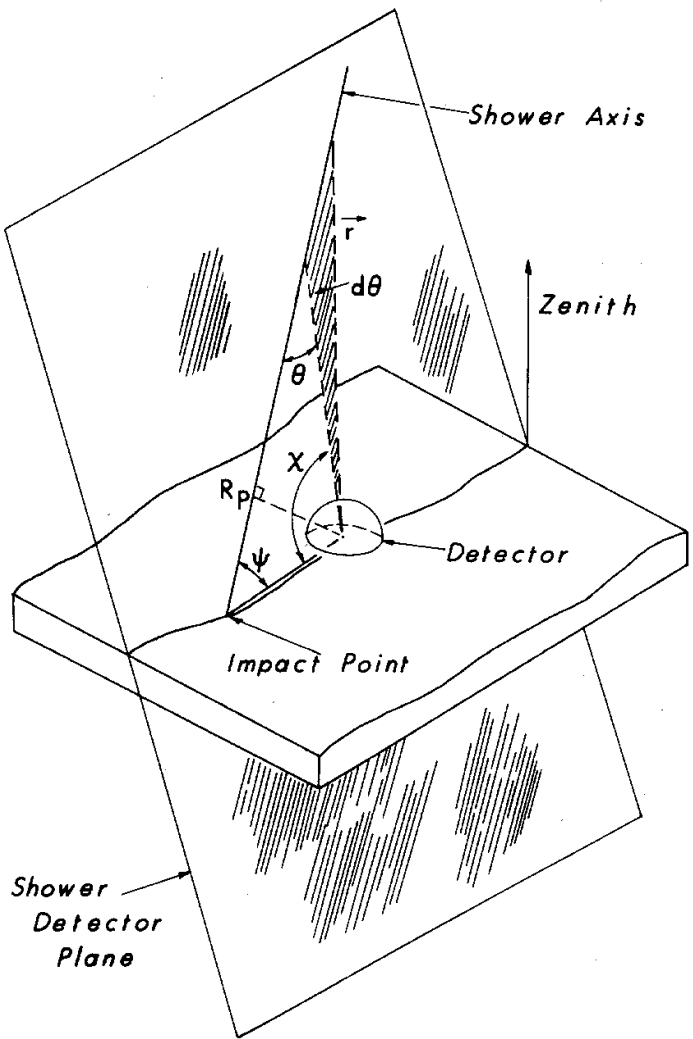

Figure 1. The shower detector plane contains the shower axis and the detector center.

An air shower that hits both detectors, SD and FD, can be fully reconstructed independently. These are called "hybrid" events and take advantage of the most important caracteristic of this experiment. With the reconstruction by two different methods a more detailed study of the EHE showers will be possible, giving a more accurate result.

The Pierre Auger Observatory will be the first EHECR hybrid detector and it will make possible a better understanding about the EHE showers detection. Besides yield its own data it is expected that PAO can help to clarify inconsistencies between existing experiments results.

\section{A. The surface detector}

The surface detector (SD) array will consist of 1600 stations spreaded on a $3000 \mathrm{~km}^{2}$ area, separated by a distance of $1.5 \mathrm{~km}$ from each other. This configuration was chosen to solve the need for good efficency to showers of $E>10^{19} \mathrm{eV}$ and statistical sampling to reconstruct the lateral distribution. Basically, the SD stations are composed of a water tank where the charged particles of EAS produce Cerenkov light, which are collected by three photomultipliers (PMTs). The tanks are made of polyethylene and the water goes inside a 
sealed bag shaping a $3.6 \mathrm{~m}$ diameter and $1.2 \mathrm{~m}$ height cylinder (Fig. 2). The roles of the liner is to prevent against water contamination, isolation of external light and to provide diffusive reflection inside the tank. The surface of the water cylinder is the sensitive area of each surface detector, $10 \mathrm{~m}^{2}$. The signal and trigger processing electronics are located on the top of the tank, powered by batteries and solar panel. The data transfer is done by radio communication to a central station. The control of the stations can be done remotely, turning it on or off when needed. Also, modifications in the software will be possible downloading the code from the central station. In this way, each SD station is a completely independent station, not needing any local interference unless some maintenance due to system fails occurs.

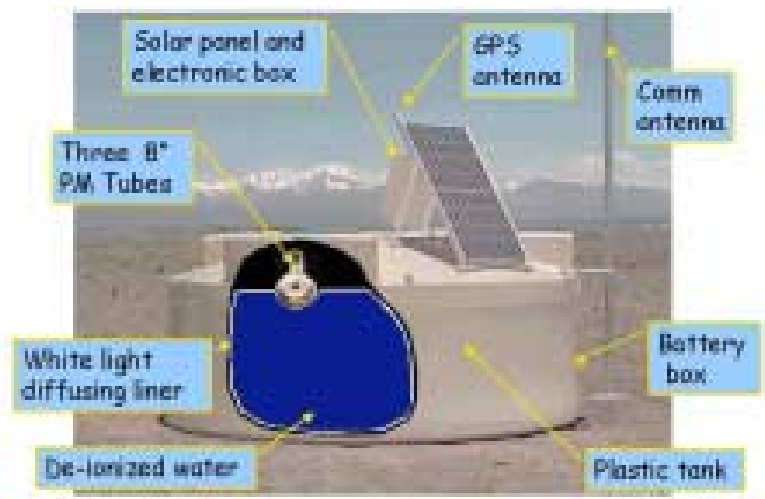

Figure 2. An surface detector station (tank) in the field. Details of the main components are shown. There will be 1600 SD stations in the southern site.

The light collected by the $8^{\prime}$ or $9^{\prime}$ (there are both sizes in use by now) diameter PMTs yield electric signals which are digitized by flash-ADC electronics. Running at sampling rate of $40 \mathrm{MHz}$, the collected data are waveforms of the signal at the output of the PMTs. This kind of information is specially useful in the reconstruction of the EAS because it brings more than the total collected charge. The analysis of the shape of the signal (risetime for instance) can help on the discrimination of muon content in the shower. Every event receives a time stamp, provided by a GPS system, possibiliting the reconstruction of the shower front direction. There are several triggers levels: level 1 and 2 are processed in the station, 3 and 4 are done at the central data acquisition.

\section{B. The fluorescence detector}

For detection of the fluorescence light there will be 30 Schmidt optics telescopes. These telescopes will be distributed in four "eyes", three at the borders of the surface detector distribution and one at the center. Each telescope unit is composed of light collector mirror, PMT camera, corrector ring lens, and associate electronics. The mirror is segmented in several pieces to allow the construction of the required surface area. The camera has a spherical surface and is composed of 440 hexagonal PMTs, distributed in 22 lines and 20 columns. Each PMT (pixel) covers $1.5^{\circ}$, performing a total camera field of view of $30^{\circ}$ in azimuth and $30^{\circ}$ in zenith angle. Each peripheral eye has six telescope, placed side by side performing $180^{\circ}$ of azimuth angle, the central one has 12 telescopes and $360^{\circ}$. Mirror electronics are encharged to digitize the PMTs signals and process the first level trigger and the Mirror PC communicates with the Eye PC to construct the eye trigger. Atmospheric monitoring systems will be running with the FD, providing data to correct the amount of light collected to the transparency of the atmosphere.

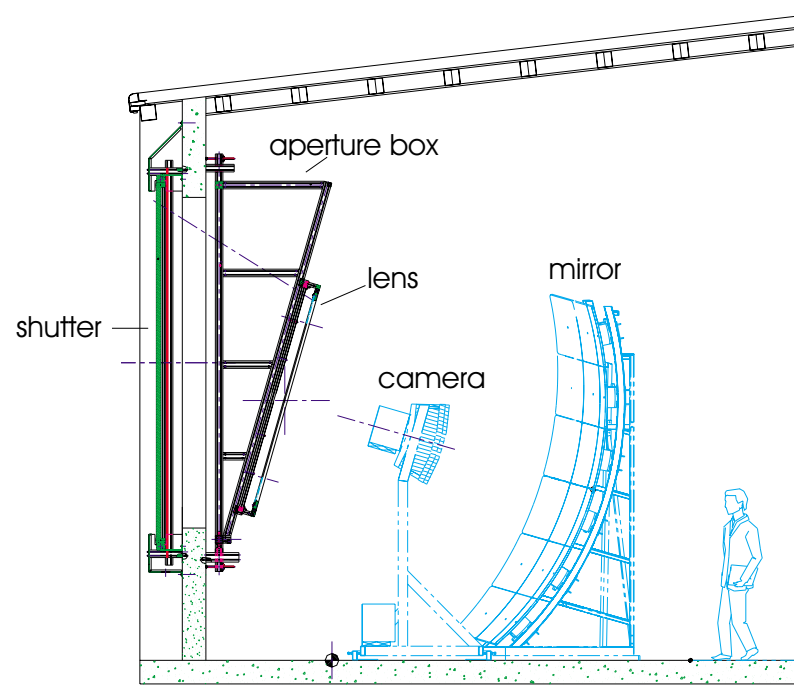

Figure 3. A telescope bay with its parts.

\section{Data acquisition and trigger}

Although the observatory uses hybrid detection technique, the detectors will not be operating always simultaneously. Surface detectors are planned to run continuously $24 \mathrm{hrs}$ a day. The fluorescence detector, though, will operate just on clear, moonless nights.

The long distance that separates each SD station makes unpractible any interconnection by wire. The data of SD stations are sent to the central data acquisition system (CDAS) by mean of wireless LAN network to a local concentrator. The concentrators are connected to the PAO backbone network, which flows the data to CDAS by microwave link. 

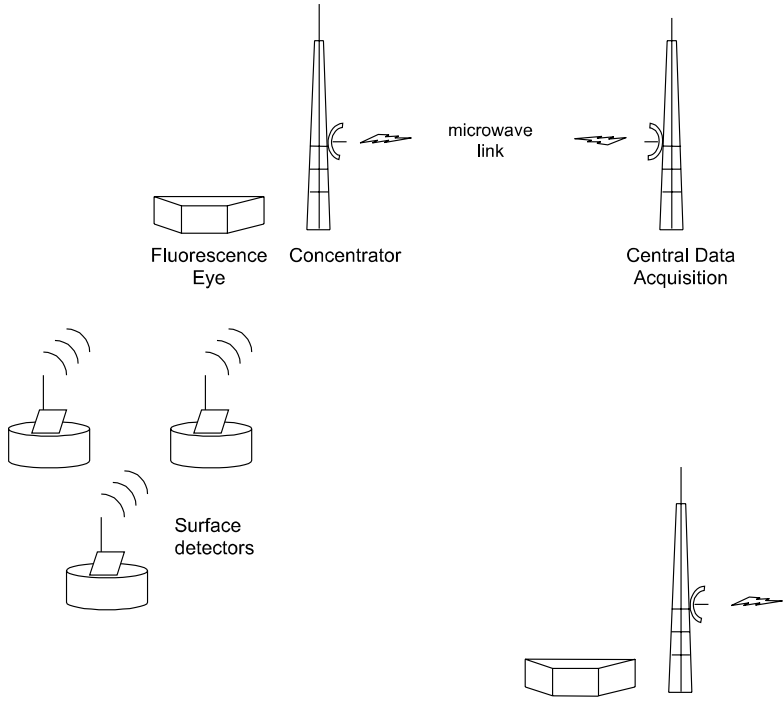

Eye

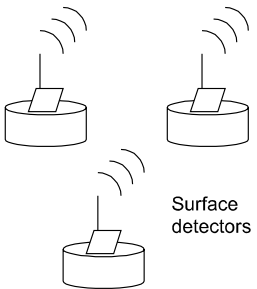

Figure 4. Diagram of the wireless communication between detectors and the central data acquisition. SD stations communicate to local concentrators, which are connected to the PAO network backbone. FD eyes are also connected to the backbone.

The SDs send a valid second level trigger (T2) to CDAS whenever the charge collected and time extension of the signal exceed a certain threshold. The CDAS looks for at least one other stations with T2 coinciding in a given time window, brodcasting a request of data in positive case. Then every triggered station sends the digitized waveforms contained in their memory buffers to CDAS.

For the FD, the trigger is performed independently of CDAS. The first level trigger is done by each pixel (PMT) and occurs when the continuously digitizing FADC system register light above the threshold of the night sky fluctuation. A second level trigger is performed when at least 4 pixels are triggered looking like a straight line. This is what is expected if a shower falls in the field of view and all the waveforms of pixels of the camera are then stored. The data are sent to CDAS by a direct connection to the network backbone.

\section{Construction status}

After a worldwide survey the two sites chosen for the installation of the PAO were Pampa Amarilla, Province of Mendoza, Argentina (southern observatory) and Millard County, Utah, USA as the northen observatory.
Besides the obvious latitude position, the main criteria to choose the sites were:

- Good climate condition, with dry atmosphere and low level of polution and light, for the operation of the FD.

- Flat land to allow wireless communications and some elevations at the border and center for installation of the FD eyes.

It has been decided that the southern site should be constructed first. The construction of the observatory has started in 2000 when the first of 1600 surface detector stations was deployed in the desert of Pampa Amarilla. Also, the Assembly Building and the first FD eye building at Los Leones mountain were constructed by that time. The $\mathrm{AB}$ is the place for the electronics/mechanics shops, storage of tools and all the construction material for the PAO. It is located in the city of Malargue and next to it is the recently finished Office Building, place of the control run and collaborators offices. Right after the central and FD communications towers were installed the firsts wireless network tests was succesfully done.

In the first phase of the project a small part of the SD array, 40 detectors, and two fluorescence telescopes will be constructed. This is the so-called Engineering Array, a prototype that will help to detect eventual technical and conceptual problems in the design of the experiment. In Fig. 5 a satellite photo of the EA site area is shown. By the time this papers was written 40 tanks was already deployed, 30 of them fully instrumented and both FD telescopes were operating.

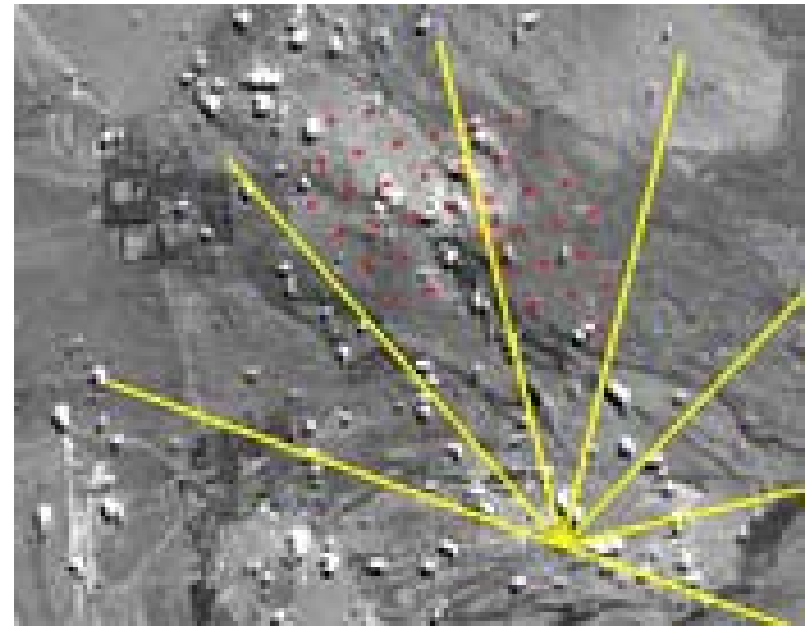

Figure 5. Engineering Array satellite photo, the diamonds marks denote the SD tanks positions. At the vertex point is the Los Leones FD eye, the lines mark the field of view of each telescope.

One of the firsts tasks in the site was to map and define the right place to deploy the tanks. They were chosen basically looking for the closest place to the projected grid point - $1.5 \mathrm{~km}$ apart to each other - with flat solid ground and easy access. The deployment of tanks 
in the field follow several steps organized in a way to optimize cost, manpower and safety:

1. Prepararation of the site. A radius of $6-10 \mathrm{~m}$ is completely cleared of vegetations to reduce the sesonal fire hazard.

2. Deployment of the tank with liner inside. The tank are brought to the field with a truck and installed using a crane.

3. Water filling. De-ionized water is produced in the water plant in the $\mathrm{AB}$ and brought to the field.

4. Installation of the PMTs, electronics and cabling.

This is the process that have been tried during the EA phase. Improvements in this methodology have to be made in order to optmize the time to construct the full array on programed schedule.

The water inside the tank has to stay clear for the transmission of the produced Cerenkov light. As PAO is programed to collect data for about 20 years, this is also the time expected the water to last. For this reason the water must be deionized and completely free from microorganisms and nutrients. After many studies it has been decided to install a water plant exclusively to fornish water for the SD tanks. It is installed in the Assembly Building and is fully operational, having produced the water to fill all the EA tanks. The water was delivered to each detector by a trailer containing 12000 liter tank (one detector capacity), pulled by a truck-like tractor on the main roads to the detectors and by agricultural-industrial tractor in difficult areas. A single hose connection has been used in an effort to prevent bacteria contamination.

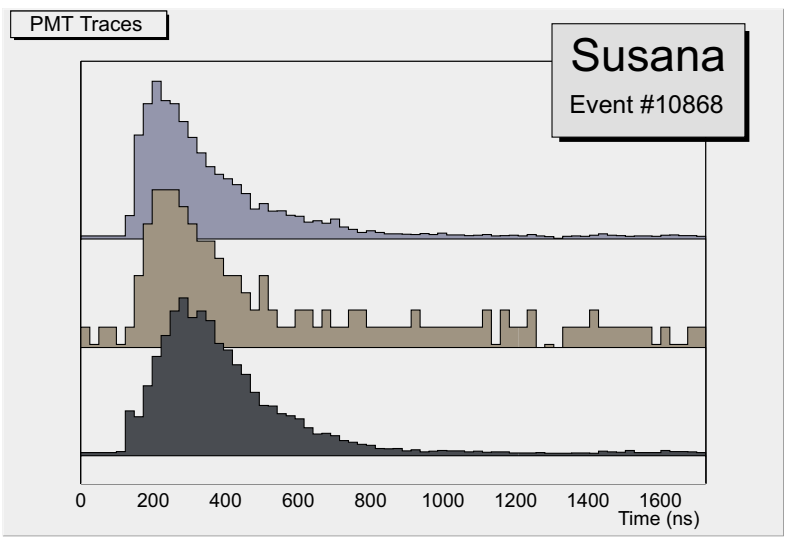

Figure 6. Digitized PMTs signals of the SD station called "Suzana". Signal amplitude (arbitrary units) by time.

Due to the wireless nature of the data acquisition and the autonomous power supply (by solar panels and batteries), the associate electronics of each SD station is a complex engineering issue. Besides the PMTs signal processing electronics it also has, power control system, radio communication module, communication interface system, GPS timing system, station computer etc. Since the firsts tanks were deployed in the field, intensive works have been done to adjust the electronic system to the real working conditions. Today there are 30 tanks in the field working and sending real data to the central data acquisition. In the Fig. 6 some waveforms from a shower event from the SD station called Suzana is shown. Even if still in the way to have a complete bug-free system, the PAO SD has proved, by its severals already detected events, that its design is successfull.

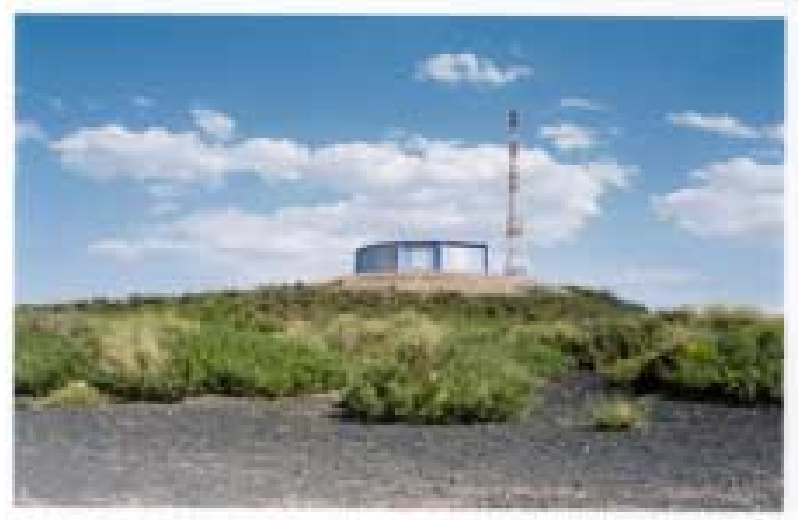

Figure 7. Los Leones FD eye building.

The fluorescence prototypes telescopes were mounted on the Los Leones site, place of the first FD eye, located at the south of the array (Fig. 7). In the EA phase two prototype telescopes was installed, their field of views overlook the sky above the 40 EA-SD stations. In this way both telescopes are able detect hybrid events and fully test the system on showers which images overlaps both telescopes. By may of 2001 the first prototype telescope was already mounted. The optical system (mirror, lenses, filters), camera, analog and digital electronics were operating. Many tests have being carried out since then and many showers have being already detected. One of these tests was done shooting laser beams in the atmosphere. The laser source was took to several different distances from the the telescopes. The laser shots are scattered in the air and it is expected that some light of semi-vertical shots goes through the telescopes. In this way an inverse shower-like structure is created. This is a good test to study the performance of the telescope in reconstructing the axis direction and light sensitivity. The results has shown that the prototypes telescopes are as sensitive as designed for and also the precison for direction determination. Fig. 10 shows an event display of one of the laser shots. There are also some hybrid events detected, they are now serving to better understand the performance of the EA. 


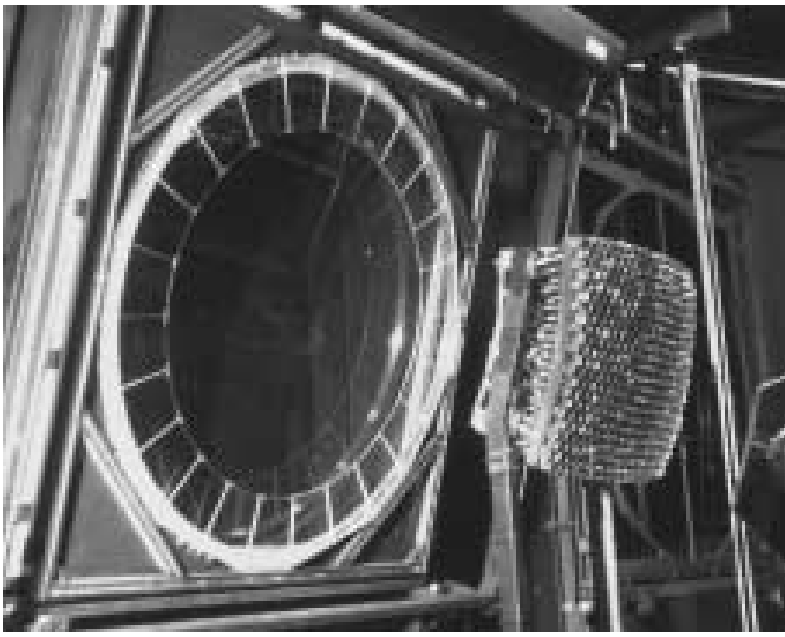

Figure 8. Detail of the camera and the aperture box with the corrector ring.
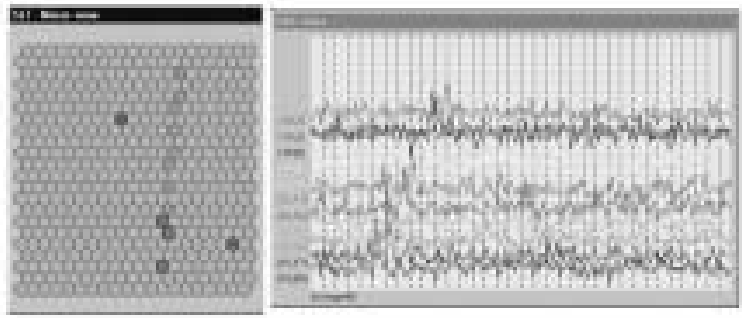

Figure 9. Camera view (left) and digitized waveform (right) of pixels of a shower event.
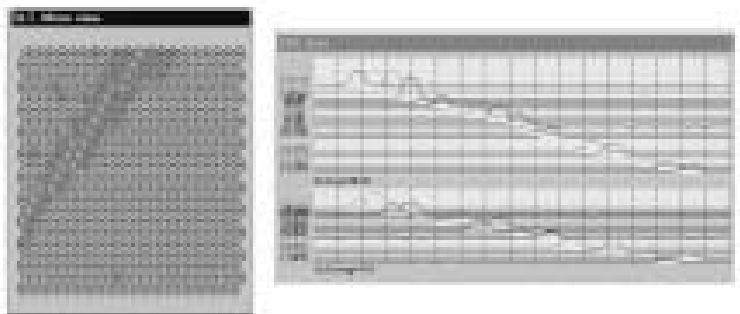

Figure 10. Camera view and digitized waveform of pixels of a laser shot event.

\section{Conclusion}

EA data taking and analysis is still going on, at the same time Pierre Auger Observatory is going forward to its pre-production phase. As result of the EA experience some parts of both detectors are being rengineered to better performance. One of them are the plastic tanks which got improvements in its design to be more robust. Also, the electronics of the SD stations are being modificate to a more definitive design using ASICs instead of PLDs. For this pre-production phase 100 new SD stations is in the way to be deployed. The FD expansion is also going on and besides the instalation of new telescopes to complete the Los Leones eye, the construction of the second eye building (El Coihueco) will start very soon. As SD, the FD expansion will have some modifications on some devices like, aperture box, lenses, shutter etc. All these modifications can be considered minor changes, not affecting the concept of the PAO detectors. The EA data analysis is showing that all the technical innovations brought into this project like auto-sufficient SD stations (solar power, radio links etc), fluorescence detectors with Schmidt optics, is successfully making possible to build a new generation of cosmic ray experiment.

\section{References}

[1] M. A. Lawrence, R. J. O. Reid, and A. A. Watson J. Phys. G 17, 733 (1991).

[2] N. Chiba, K. Hashimoto, and N. Hayashida et al, Nuclear Instr. and Methods A311, 338 (1992).

[3] M. Nagano, M. Teshima, Y. Matsubara, H. Y. Day, T. Hara, N. Hayashida, M. Honda, H. Ohoka, and S. Yoshida, J. Phys. G 18, 423 (1992).

[4] R. M. Baltrusaits et al, Nuclear Instr. and Methods A240, 4101985.

[5] D. J. Bird, S. C. Corbato, and H. Y. Dai et al., Astrophys. J. 424, 491 (1994).

[6] A. M. Hillas Ann. Review of Astronomy and astrophysics 22, 425 (1984).

[7] E. Fermi, Physical Review 75, 1169 (1949).

[8] K. Greisen, Physical Review Letters 16, 748 (1966).

[9] G. T. Zatsepin and V. A. Kuz'min JETP Letters 4, 78 (1966).

[10] T. K. Gaisser Cosmic Ray Physics, Cambridge University Press, 14.4, 202 (1990). 